Cinémas

Revue d'études cinématographiques

Revue d'études cinématographiques

Journal of Film Studies

\title{
Le cinéma d'animation et ses thanatomorphoses (fragments sur le monstre, la charogne, le montage et l'animation)
}

\section{Dick Tomasovic}

Volume 13, numéro 1-2, automne 2002

Limite(s) du montage

URI : https://id.erudit.org/iderudit/007960ar

DOI : https://doi.org/10.7202/007960ar

Aller au sommaire du numéro

Éditeur(s)

Cinémas

ISSN

1181-6945 (imprimé)

1705-6500 (numérique)

Découvrir la revue

Citer cet article

Tomasovic, D. (2002). Le cinéma d'animation et ses thanatomorphoses (fragments sur le monstre, la charogne, le montage et l'animation). Cinémas / Revue d'études cinématographiques, 13(1-2), 143-164.

https://doi.org/10.7202/007960ar
Résumé de l'article

Le rôle et les limites du montage dans le cinéma d'animation sont ici reconsidérés. Il ne s'agit plus de l'entendre comme un « simple » travail d'assemblage de plans, d'associations d'éléments épars, de collage de morceaux divers, de constitution d'un grand corps monstrueux, mais bien de l'étudier comme un processus permanent de gestion de passages, de modulations d'une image à l'autre, de transformations d'éléments inanimés, de transmutations de figures fixes, de métamorphoses de corps inertes. En ce sens, le projet du cinéma d'animation n'est pas autant celui, fantasmatique, du don de vie que celui, fantasmagorique, d'animation de la mort. 


\section{Le cinéma d'animation et ses thanatomorphoses (fragments sur le monstre, la charogne, le montage et l'animation)}

\section{Dick Tomasovic}

\section{RÉSUMÉ}

Le rôle et les limites du montage dans le cinéma d'animation sont ici reconsidérés. Il ne s'agit plus de l'entendre comme un "simple" travail d'assemblage de plans, d'associations d'éléments épars, de collage de morceaux divers, de constitution d'un grand corps monstrueux, mais bien de l'étudier comme un processus permanent de gestion de passages, de modulations d'une image à l'autre, de transformations d'éléments inanimés, de transmutations de figures fixes, de métamorphoses de corps inertes. En ce sens, le projet du cinéma d'animation n'est pas autant celui, fantasmatique, du don de vie que celui, fantasmagorique, d'animation de la mort.

\section{ABSTRACT}

The role and limits of editing in animation film are reconsidered here. It is no longer a matter of perceiving this practice as "simply" assembling shots, associating random elements, linking diverse pieces and creating a monstrous body, but rather, it is presently regarded as a continual process of managing transitions, shifting between images, transforming inanimate objects, transmuting immobile elements and transfiguring inert beings. Thus, the animation film's purpose is less phantasmal, to be a giver of life, as it is phantasmagoric, to be an animator of death. 
I.

Les mouches bourdonnaient sur ce ventre putride,

D'où sortaient de noirs bataillons

De larves, qui coulaient comme un épais liquide

Le long de ces vivants haillons.

Tout cela descendait, montait comme une vague,

Ou s'élançait en pétillant;

On eût dit que le corps, enflé d'un souffle vague,

Vivait en se multipliant.

Baudelaire, «Une charogne» (extrait), Les Fleurs du mal.

II.

On dit souvent que le cinéma d'animation est privé, de par la nature particulière de son dispositif, de l'opération fondamentale du montage. C'est là, encore une fois, une de ces assertions emportées et approximatives dont l'animation se voit régulièrement victime. Comme toute œuvre cinématographique, le cinéma d'animation mobilise quantité de sons et d'images qu'il est nécessaire d'agencer et de combiner. Ces multiples «arrangements " vont de la sélection des éléments utiles parmi les rushes (donc de l'abandon, souvent douloureux, d'un certain nombre d'éléments tournés), et de leur assemblage dans un nouvel ordre (le fameux ours, premier montage-martyr) jusqu'au choix définitif de la longueur des segments et de l'établissement précis de leurs raccords ${ }^{1}$. Le montage n'est donc en aucun cas une triste opération technique, mais bien un principe cinématographique régissant l'organisation des divers éléments filmiques à l'aide de nombreux effets syntaxiques purement rythmiques ou pleinement sémantiques, effets de liaison ou de rupture, de linéarité ou de ponctuation, d'alternance ou de contrepoint, etc. : tout cela est bien connu.

Comme le fait remarquer Vincent Amiel (2001, p. 1), le cinéma s'inscrit dans un vaste courant de création artistique né au XIX $^{e}$ siècle (peinture, sculpture, musique, bande dessinée), caractérisé par la mise en valeur de l'idée de fragments possiblement associés selon d'inédites logiques. Ainsi, la notion nous est acquise, le montage ne se borne pas à une opération technique, 
mais se révèle également un principe de création, l'acte même de la conception de l'œuvre au moment où sont associées ses images éparses. D’emblée, on voit mal pourquoi le cinéma d'animation se verrait privé de ce moment privilégié de création, d'autant que l'hétérogénéité des images, produites ou empruntées, et leur morcellement en une infinité de fragments, représente une des constantes de ce type de cinéma. Cependant, l'opération technique du montage d'un film d'animation connaît certaines modalités qui lui sont propres. Les animateurs Borivoj Dovnikovic (2000, p. 170-171) et Jacques Drouin (1982, p. 16-18) ont détaillé le travail particulier du monteur de film d'animation. Tous deux identifient le propre du montage en cinéma d'animation avec le fait qu'il intervient dès le début $\mathrm{du}$ film, donc qu'il est antérieur au moment où il intervient dans le cinéma traditionnel, qui le considère comme l'une des dernières étapes du processus cinématographique ${ }^{2}$. Le cinéma d'animation dans son ensemble ne connaît pas, ou nettement moins, les étapes distinctes du parcours de fabrication d'un film traditionnel. Il est un processus continu, une aventure ininterrompue précédant de beaucoup la réalisation. Le film d'animation est le film prémédité par excellence.

III.

Les raccords sont précisément autour du point de montage (le moment précis où les plans se succèdent) un moyen de jouer sur la continuité des plans. "Raccorder", c'est faire en sorte, comme le terme l'indique, que le cut ne soit pas ressenti comme une rupture définitive et radicale, mais comme l'occasion d'une couture, qui permet d'assembler des morceaux différents avec la plus grande discrétion. Il s'agit de camoufler la césure, d'en effacer l'impression, tout en conservant la qualité d'articulation qui est au principe des changements de plan (Amiel 2001, p. 18).

Alors que Vincent Amiel décrit l'acte essentiel du montage, c'est-à-dire couper et coller (ou en un mot: raccorder), la conception du montage dans le film d'animation se fait beaucoup plus fuyante. Aucune des opérations du montage classique - le 
point de montage, la continuité des plans, le cut comme couture, le camouflage de la césure, l'articulation du changement de plan - ne rencontre ici la réalité du film d'animation, où la notion de plan elle-même est caduque (cette notion ne pouvant que renvoyer ici à une série d'images dont la vague et subjective cohérence seule détermine l'unité syntaxique). Non, vraiment, le cinéma fabriqué une image à la fois ne recourt ni au découpage ni au collage, et ses images ne se raccordent pas, elles sont construites de concert, l'une étant conçue inévitablement comme la suite de l'autre. Quiconque s'est déjà risqué dans un atelier de dessin animé sait que les animateurs ne travaillent une image que par rapport à une autre image. Incroyable exercice de dessin, d'imagination rigoureusement réglée, qui consiste dans une tension perpétuelle à soulever à chaque minuscule coup de crayon les feuilles et les calques pour vérifier tout à la fois la justesse des superpositions et les infimes décalages entre images presque siamoises appelées à s'enchaîner parfaitement. Formidable travail de la distinction dans le semblable. Prodigieuse et inquiétante loi de l'enchaînement des images, dans son sens le plus "enchaînant", appliquée sous le règne absolu et monomaniaque de leur défilement idéal. L'image comme punie, condamnée à se répéter mais en variant légèrement. L'impitoyable règle est la même pour tous les studios d'animation, quelle que soit leur technique de travail: les images n'existent qu'en fonction les unes des autres.

On l'aura compris, s'il y a une spécificité du travail et de la conception du montage dans le cinéma d'animation, si ce dernier comporte une réelle différence sur ce point avec le cinéma "non animé", elle ne se situe pas, à mon sens, dans le moment où intervient le montage (car il s'agit dans l'ensemble du même travail d'élaboration: que faut-il mettre et dans quel ordre fautil le mettre) ni dans sa technicité (le travail de finition est globalement le même: ne pas accrocher l'œil ou l'oreille, ou bien tout faire au contraire pour l'accrocher le plus violemment possible), mais dans l'approche esthétique qui domine ce travail de patiente élaboration, de montage de l'œuvre depuis son commencement, et qui prend une tout autre dimension dans le film d'animation: spécificité qui favorise l'émergence d'une poétique 
singulière, qui sollicite les images autant que leurs intervalles, qui s'élabore dans la matière même du film, dans le dispositif filmique même, qui œuvre dans et à partir de son flux temporel, et qui est nulle autre que celle de la métamorphose. Il ne faut pas s'y tromper: cette spécificité ne peut être réduite à une figure de construction isolée ni non plus dominante, dotée d'enjeux esthétiques certes reconnus importants, toutefois décrite comme simplement récurrente, périodique ou subite. Si l'on veut, un "effet" par ailleurs fascinant du cinéma d'animation. À mon sens, la métamorphose constitue la figure de construction fondamentale et invariable du cinéma d'animation, dictée précisément par ce que nombre d'auteurs ont appelé la nature synthétique du processus d'animation; elle est le mode même de fonctionnement de ce processus; elle m'apparaît donc comme omniprésente.

Changer un dessin, même d'une manière infime, c'est inévitablement, lors de la projection, l'amener à se transformer. En ce sens, si certaines époques ou certains corpus se sont montrés davantage enclins à démontrer cette puissance de la métamorphose, il n'en est pas moins vrai que celle-ci se révèle, tout au long de l'histoire des images animées, comme leur condition permanente. Alexandre Alexeieff (1966, p. 19) l'avait tout à fait compris lorsqu'il a établi l'importance de ce qu'il nommait "l'autre moitié du film», que Norman Mclaren appelait pour sa part, dans sa fameuse définition de l'animation, le "between each frame", dont l'existence virtuelle ne peut qu'échapper à la perception lorsqu'on examine une bande à la main, mais qui permet de transformer un vulgaire changement de position cardinale en mouvement lorsqu'on la projette. Alexeieff devait d'ailleurs inventer un formidable instrument à métamorphoses, une nouvelle technique d'animation, celle du fameux écran à épingles, dont le seul exemplaire encore utilisé aujourd'hui se trouve en possession de Jacques Drouin de l'Office national du film (Canada), un dispositif qui joue de la lumière latérale et des ombres qu'elle fait porter sur une surface couverte d'épingles pouvant plus ou moins s'enfoncer, faisant apparaître ainsi des motifs tout en nuances ou en contrastes ${ }^{3}$. En raison de la particularité du dispositif, et de l'immense 
travail de manipulation qu'il exige, l'animateur est appelé à modifier très progressivement l'image, épingle par épingle a-t-on envie d'écrire, plutôt que de lui en substituer une autre. Mais d'autres techniques d'animation nécessitent la transformation d'une forme en une autre. C'est notamment le cas de l'approche par la pâte à modeler. Si l'on en croit un de ses maîtres, Peter Lord, on trouve dans cette approche une véritable énergie propre à l'animation ${ }^{4}$. Michael Frierson, historien de la «clay animation", louange également la pâte à modeler, en raison de sa plasticité et de son pouvoir de transmutation des formes. La pâte donne lieu à de multiples transformations incongrues, détruisant l'idée même d'une forme statique, stabilisée. Pour cette raison, elle fait de l'animation une expérience puissamment fantasmatique et métaphorique, entraînant le spectateur loin de ses repères, loin du monde "naturel» dans lequel il vit, dans lequel la plupart des choses et des événements quotidiens sont clairement arrêtés et définis ${ }^{5}$.

Ces rencontres de formes, ces passages d'une image à l'autre, si elles sont possibles dans le cinéma "non animé» par le recours à un certain type de montage (parallèle, de correspondance ou intellectuel, peu importe son appellation), constituent l'ordinaire de l'unique et inéluctable type de montage qui soit en cinéma d'animation. Georges Sifianos, qui s'est intéressé à la psychologie expérimentale, rapporte que toute image que l'on copie plusieurs fois tend à se modifier en se simplifiant, donc que la dernière image copiée est différente de la première. Pour cet auteur, c'est là le processus même de la métamorphose ${ }^{6}$. Ainsi, dans le cinéma d'animation, les images se succèdent moins qu'elles ne s'enchaînent, se confrontent moins qu'elles ne se fondent les unes dans les autres. Bref, la métamorphose fait se rencontrer les formes et ne leur permet plus de se refermer sur elles-mêmes. Elle apparaît comme le type de montage, peut-être étrange, mais premier en cinéma d'animation (ce qui n'empêche d'aucune manière le recours à d'autres formes de montage par la suite, assemblant des unités comparables à des plans par leur homogénéité) - comme s'il s'agissait dans un premier temps, le terme vaut ce qu'il vaut, de micromontage. Ainsi, l'animation ne se prive point de l'approche frankensteinienne ${ }^{7} \mathrm{du}$ montage 
cinématographique, du jeu du coudre, de l'assemblage des différentes pièces de l'ensemble. Bien au contraire, la métamorphose rend l'événement du raccord omniprésent, elle le systématise au point qu'elle force son principe jusque dans ses retranchements, là où il s'ouvre sur d'infinies discontinuités, parce que comme le souligne Pierre Hébert, dans le cinéma d'animation, le temps est fabriqué ${ }^{8}$.

\section{IV.}

Modeling, film d'animation datant de 1923, est un épisode de la série populaire Out of the Inkwell, réalisée et produite par les frères Fleischer entre 1916 et $1927^{9}$. Le film, combinant malicieusement techniques d'animation et prises de vues réelles, interroge justement l'existence d'un montage propre à l'animation. Il recourt non seulement à l'animation de dessins, mais aussi à celle de volumes. Modeling, en conjuguant ces deux techniques d'animation, qui elles-mêmes entretiennent de savantes relations avec les prises de vues réelles, se présente comme une œuvre hybride, comme une hydre à plusieurs têtes.

Tous les épisodes de la série obéissent à un canevas précis. La main d'un animateur (celle de Max Fleischer) crayonne la forme de Koko le clown, un personnage aussi maladroit qu'acrobatique, aussi nigaud que gouailleur. Le dessin de Koko s'anime et entre aussitôt en interaction avec le monde de l'animateur. La drôlerie des situations provient bien sûr de l'effronterie du clown face à son créateur, mais surtout de la perméabilité soudaine d'un monde physique, réaliste, à celui, fantasmagorique, du dessin animé. Koko, par une série d'actes saugrenus, a tôt fait de perturber le monde réel et l'animateur doit alors redresser la situation en contraignant le petit clown à regagner son encrier. Le procédé narratif est donc itératif, et certaines séquences dessinées sont reproduites, pour d'évidentes raisons économiques. Modeling ne s'écarte aucunement de ces normes.

Le film s'ouvre sur quelques dessins étalés sur une table. Une main et un stylo sont au travail, traçant quelques petites formes circulaires, des bulles, sur une feuille de papier d'une blancheur éclatante. La main soulève la feuille, puis la penche. Les petits ronds s'animent aussitôt et forment des sortes de gouttes qui 
s'alourdissent et menacent de tomber de la feuille. L'animateur les recueille alors avec son stylo. Il s'agit là d'un bref prologue totalement emblématique révélant dès le départ le procédé de construction itératif de la série. Une goutte s'échappe soudain du stylo, entame quelques pirouettes, se décompose en un cortège de gouttelettes qui vont enfin donner forme à Koko le clown, qui commence dès lors à s'animer, puis à saluer de la main son créateur mais aussi le spectateur. Pendant que Max Fleischer rend les postures de son pitre plus dynamiques, un gentleman portant un haut-de-forme et arborant un nez gigantesque entre dans l'atelier. Un deuxième animateur (il s'agit de Roland Crandall, participant assidu de la série) le reçoit et l'invite à s'asseoir devant lui. Crandall dévoile alors la sculpture d'une tête dont le visage reproduit celui du gentleman. Ce dernier prend une pose et Crandall se met au travail. Du côté de Max Fleischer, Koko continue d'être malmené. La caméra revient à Crandall: le gentleman lui reproche, dans un approximatif champ-contrechamp, la trop grande ressemblance entre son visage et sa reproduction (la taille du nez, peu flatteuse, pose problème). Crandall, défendant son travail, appelle Max qui, avant de rejoindre son collègue, greffe des patins à glace aux pieds de Koko et le place au centre d'un décor hivernal. Alors qu'une discussion s'engage entre les trois hommes, Koko prend vie: pour commencer, il perd l'équilibre, mais peu à peu il parvient à se contrôler au point de bientôt pouvoir nous offrir une jolie démonstration de sa toute nouvelle maîtrise du patinage. Il s'éloigne à toute vitesse et disparaît soudainement pour réapparaître tout aussi vite comme s'il était doté d'un pouvoir d'ubiquité, comme si la notion de distance ou de localisation spatio-temporelle n'existait pas dans l'espace particulier de la page blanche (son vide semble permettre la libre circulation des éléments, la communication globale des points cardinaux entre eux). Plus loin, les animateurs et le gentleman discutent toujours du nez de la sculpture. Sur la glace, Koko dessine avec ses patins une caricature du visage du gentleman. Celui-ci entraperçoit l'affront, mais le dessin s'évanouit aussitôt et Koko doit alors affronter un ours taquin qui lui vole son chapeau. Une série d'évènements absurdes et ahurissants se 
produisent alors entre Koko, l'ours, une vache et une maison. Koko finit par écraser l'ours avec une gigantesque boule de neige qu'il sculpte ensuite en une nouvelle caricature de la tête du gentleman. Cette fois, le modèle surprend les clowneries de Koko et s'en plaint aux animateurs. Fleischer arrache un morceau de glaise à la sculpture de Crandall et le lance vers la planche à dessin. Koko est touché à la tête, mais ne tarde pas à renvoyer la boule de glaise en direction de ses adversaires. Le gentleman est touché à l'œil. Profitant de la situation, Koko quitte la feuille de papier pour rejoindre le monde «réel»: il patine un temps sur la table, puis sur le tapis de sol, pour atteindre enfin le pied de la sculpture et l'escalader. Crandall et le gentleman reprennent leur tâche, mais soudain le nez de la sculpture (dans lequel Koko s'est dissimulé) commence à s'agiter en tous sens, comme un serpent se tortillant, et quitte la sculpture pour ramper au sol ou se dandiner joyeusement. Les deux animateurs et le gentleman parviennent à capturer le petit morceau de glaise, mais Koko s'en est déjà échappé. Le gentleman, furieux, décide de quitter l'atelier, non sans lancer une boule de glaise aux deux animateurs. Koko réintègre au plus vite sa feuille de dessin par un trou dans le papier (selon le dessin, un trou dans la glace) qui permet le passage d'un monde à l'autre. Courroucés et menaçants, les deux animateurs se rapprochent de la table à dessin. Koko prend peur et préfere regagner l'abri de l'encrier. Le dernier plan du film montre la main de Fleischer qui renverse le récipient d'encre et laisse se répandre le sombre liquide en une longue tache désolante et dépourvue de toute la magie dont elle était jusque-là investie.

On l'aura compris en lisant cette description, le film repose sur un montage alterné entre, d'une part, les facéties de Koko le clown sur sa feuille de dessin et, d'autre part, la discussion entre les deux animateurs et le gentleman, personnages réels. Les espaces animé et réel sont amenés à se rencontrer dans leurs différents points de contact: la main de Fleischer dessinant Koko, les moqueries de Koko dessinant le gentleman; la boule de glaise lancée sur Koko par Fleischer; le clown qui renvoie la boule sur le gentleman; etc. Le moment le plus fantastique dans la rencontre de ces deux mondes est celui non pas de l'intrusion 
parfois fulgurante d'un élément animé dans l'univers réel et vice versa, mais de la transformation du monde réel en monde animé lorsque Koko fuit sa planche à dessin et traverse l'espace réel de l'atelier pour se dissimuler dans le nez de glaise de la sculpture du gentleman ${ }^{10}$. Dès lors, le film change brusquement de logique: la démarcation entre les espaces animé et réel n'est plus maintenue et cette confusion effraie même les animateurs (Crandall et Fleischer, apeurés, se cachent lorsqu'ils voient le nez de la statue s'animer $\left.{ }^{11}\right)$. Le beau principe d'alternance est oublié, le montage des plans est désormais incertain. Les espaces ne sont plus en vis-à-vis, ni même adjacents, mais semblent s'enchevêtrer confusément: la planche à dessin semble appartenir au monde «réel» (plus rien ne bouge à présent sur les feuilles de papier), mais ce monde réel est lui-même animé de l'intérieur (la sculpture, pivot du film depuis son début, structure l'espace et permet de décerner aux personnages et aux éléments leurs places respectives).

Ainsi, alors que le film se donnait dans un premier temps comme un modèle de montage alterné ${ }^{12}$ (les deux univers évoluant chacun de leur côté pour se rencontrer en bout de ligne), le voilà qui bascule soudainement dans le désordre le plus complet. Cette situation chaotique, qui va bouleverser la diégèse (tout le monde perd son calme) autant que notre réception (nos repères sont moins sûrs), est bien sûr le fait de Koko, mais non tant de son rôle de personnage que de son état de personnage animé qui engendre un nouveau type de montage dans une structure pourtant préétablie (l'animation d'un côté, constituée de scènes généralement longues, se déroulant dans un décor unique, sans effet de découpage, comme s'il s'agissait d'un plan séquence facilement gérable, et de l'autre côté un découpage rigoureux des séquences en prises de vues réelles). La métamorphose s'est infiltrée dans la série photographique, le dessin a supplanté le plan. L'animation s'est répandue, a contaminé le monde qui lui était adjacent. Le petit personnage animé a transformé le décor réaliste (les meubles de l'atelier prennent l'aspect d'un celluloïd d'arrière-plan), la conception de l'espace (les polarités si bien définies tout au long $\mathrm{du}$ film ${ }^{13}$ se sont évanouies) et la perception des corps (l'inerte sculpture devient 
une figure moqueuse à son tour). La perturbation cesse quand Koko réintègre la planche à dessin et que la métode d'assemblage de départ est restaurée, opposant deux types de montage clairement définis (la métamorphose du dessin animé et les règles de raccords - de regard ou dans l'axe de la caméra — des séquences en prises de vues réelles). La composition même du dernier plan (la main de l'animateur, réaliste, renversant l'encrier pour répandre son contenu sur l'espace blanc de l'engendrement fantasmagorique; le contrôle quasi divin de l'animateur, à la fois perdu et retrouvé en noyant l'espace même de sa création) confirme le retour à l'ordre des choses en faisant mine de supprimer toute nouvelle possibilité d'animation. Ainsi la métamorphose, même lorsqu'elle ne se manifeste pas en tant que figure de construction, même lorsqu'elle est soumise à une méthode d'assemblage qui l'englobe, œuvre à la déstabilisation de l'image, qu'elle ouvre inéluctablement sur l'inconnu ou, en tous cas, sur l'incertain.

V.

L'incertain, d'ailleurs, est manifeste dans toutes les images relevant de l'animation - à commencer par celles des pionniers, Emile Cohl ou Stuart Blackton - mais aussi du registre fantasmagorique même. La lanterne magique d'ÉtienneGaspard Robertson avait bien compris que toute pratique de la métamorphose est incontestablement occulte, macabre, sépulcrale $^{14}$. La passion de Robertson pour les images animées ne réside pas ailleurs que dans leur absence même de corporalité, dans leur état fantomatique. Très vite, il lui fut évident qu'animer des images, c'était jouer avec des apparitions et des disparitions, que c'était en somme faire parler les morts. Il n'en fallut pas plus pour que la nécromancie s'installe au cœur de son travail: en janvier 1798 paraissait dans les gazettes la première annonce de sa fantasmagorie décrite comme une série "d'apparitions de spectres, de fantômes et de revenants tels qu'ils ont dû et pu apparaître dans tous les temps, dans tous les lieux et chez tous les peuples ${ }^{15}$ ". Bien sûr, la métamorphose robertsonienne constitue une figure de l'espace (la rencontre en un même lieu de deux corps différents, le premier s'oubliant au 
profit du second, s'y fondant pleinement pour former un temps un corps inédit, un de ces hybrides monstrueux, aux jambes d'écailles et aux ailes de chauve-souris ${ }^{16}$, qu'affectionnait particulièrement Robertson), mais elle constitue surtout une figure du temps, celle du travail même de la mort. La contamination des espaces que provoque Koko révèle sa nature: le buste du gentleman, œuvre potentiellement mortuaire puisque vouée à la postérité, s'anime soudain; son visage figé est pris d'étranges contorsions, puis d'un grouillement sous-cutané (Koko se déplace à l'intérieur de la glaise), enfin le nez se déforme, se tortille et se sépare du reste du visage, comme un morceau ignominieusement gâté tombant d'un ouvrage en lambeaux ${ }^{17} .$. Il faut penser ici à ces animateurs extrémistes, Ladislas Starewitch, Jan Svankmajer ou Manuel Gomez, qui n’ont pas hésité à animer des corps morts (insectes naturalisés ${ }^{18}$, matières organiques détériorées, viandes rouges). La putréfaction n'estelle pas la métamorphose ultime des corps?

VI.

Le monstre le plus répugnant et le plus agressif qui métamorphose les choses les plus belles en horreurs insupportables, c'est bien le cadavre en voie de décomposition. La putréfaction de la chair, signe absolu de la mort, inaugure en effet la phase la plus dramatique du processus thanatique et devient le lieu privilégié où s'amalgament les fantasmes les plus irréductibles; aussi est-ce à son propos que les rites funéraires prennent tout leur sens (Thomas 2000, p. 472).

D'après l'anthropologue Louis-Vincent Thomas, la putréfaction est malgré sa mauvaise odeur repoussante moins comme réalité objective qu'en raison des fantasmes qu'elle déchaîne. La pourriture, la décomposition du corps, la déliquescence des chairs crient la dissolution de l'être et de son individualité, démentent le sens même de la vie. Et c'est moins la saleté de la putréfaction qui repousse que la souillure qu'elle représente, ou les risques de contagion qu'elle comporte pour les vivants, qui n'ont dès lors de cesse de ritualiser pour cacher, contrôler ou 
supprimer la putrescence ${ }^{19}$. L'une des idées maîtresses développée dans son ouvrage consacré au cadavre ${ }^{20}$, et sur laquelle il revient dans un ouvrage postérieur ${ }^{21}$, repose sur la relative noblesse des parties dures qui apparaissent après la mort (la blancheur du squelette) par rapport aux parties molles, victimes de la putréfaction. Le squelette s'est ainsi affirmé, à travers les nombreuses images culturelles ou artistiques de la mort au fil des siècles comme le symbole de la Mort ou du mort. Le phénomène de la thanatomorphose s'en est trouvé occulté, oublié, alors qu'il est le processus, l'acte même, le travail de la mort.

Les signes annonciateurs de la défaite sont les lividités. Le sang qui ne circule plus obéit aux lois de la pesanteur et les divers liquides de l'organisme se déposent aux parties les plus déclives du cadavre en relation avec la position de celui-ci. [...] Mais le pire ne va pas tarder, je veux parler du processus de charognisation, la putréfaction, phase dramatique et scandaleuse $\mathrm{du}$ processus thanatique, lieu privilégié où s'amalgament nos fantasmes les plus irréductibles. [...] Il n'est en effet guère de spectacle plus affligeant que celui du cadavre entré en pourriture, pour peu qu'il se vide prématurément. Il pue. [...] Il transforme les chairs redevenues molles et flasques en masses fétides, diffluentes puis pultacées [...]. Privé de tout métabolisme cellulaire qui assurait l'autonomie du corps vivant, le cadavre s'abandonne tout entier aux mécanismes de désintégration-récupération (Thomas 1991, p. 308-309).

À la lecture de ces quelques lignes, forcément pénibles mais qui expriment sans détour la terrible désolation qui frappe le corps des décédés ${ }^{22}$, la mort semble bien dotée d'un étrange pouvoir de métamorphose du cadavre, qu'elle traite comme une matière privilégiée, comme le support d'une série d'événements inusités, comme le territoire d'un nouveau cycle d'activités. La mort s'étale dans la durée ${ }^{23}$. Et la vie constitue une morbidité évolutive, l'individu étant porteur lui-même de mort, la contenant en quelque sorte: "Les vers qui mangent les cadavres ne viennent pas de la terre, mais de l'intérieur du corps, de ses 
liqueurs naturelles» (Ariès 1975, p. 39-40). La mort est créatrice de forme. Cette inscription dans le temps du changement des formes nous ramène en définitive au dispositif cinématographique et me rappelle cette phrase de Bazin, l'une des rares consacrée au cinéma d'animation, le théoricien y évoquant les films de Cohl, Fischinger, McLaren et Len Lye: "L'animation n'est pas alors pure transformation logique de l'espace, elle est de nature temporelle. C'est une germination, un bourgeonnement. La forme engendre la forme sans jamais la justifier" (Bazin 1994, p. 198).

\section{VII.}

L'ONF et particulièrement le studio français de l'ONF ont toujours, on le sait, fait la part belle à l'expérimentation en animation et encouragèrent le développement de projets où des propriétés fondamentales de l'animation, telle la métamorphose, étaient mises en évidence ${ }^{24}$. Le travail de Suzanne Gervais, depuis le début des années 1970, s'inscrit pleinement dans cette perspective. Son premier film, dessiné en 1971 et intitulé Cycle, est composé d'une longue série de transformations ayant pour thématique l'insertion de l'individu au milieu de son univers. D'une fausse simplicité, cette rêverie animée s'exprime par les métamorphoses continues de traits dessinés sur du papier blanc. La transformation du titre Cycle fait du reste partie du dispositif. Les traits des lettres s'espacent, puis s'étendent pour donner lieu à une nouvelle forme, indescriptible. Se désagrège alors cette forme. Une multitude de grains épars, grouillant de toutes parts, se rassemblent en spirale et donnent naissance au corps, quelque peu schématisé, d'une femme. De ce corps se distingue un visage, d'où se dégagent de nouvelles spirales, desquelles émerge encore une silhouette, emportée par le vent, croisant un cercle qui lui sert de planète et à laquelle elle se fond. Les traits s'enchevêtrent, une forêt surgit. Il devient désormais presque impossible de définir les événements. Le travail purement pictural et cinétique touche à l'indicible. Ici et là, on voit une silhouette se transformer en fleurs, puis en oiseaux, puis redevenir ce corps féminin des premières secondes du film, avant de devenir cheval, vagues, végétation luxuriante, poissons, etc. Le film 
appartient au spectateur, chacun interprétera les formes selon ses propres références. Un jeu permanent d'allers et retours entre les niveaux macro et micro-environnementaux rythme le film. Le titre réapparaît enfin, suivi d'un bref générique, amené luimême à se déformer pour reformer le corps de la jeune femme du début du film. Composé d'un seul plan-séquence voudraiton écrire, le film recourt à un montage permanent de formes, aussi exigeant en fait de précision qu'insolite dans les rapprochements qu'il occasionne, donnant le sentiment d'une totale osmose entre tous les éléments présentés. Cette interpénétration des formes, leur profond enchevêtrement, leurs boucles, rend obsolète le clivage vie-mort: la forme naissante est aussi une forme mourante, l'apparition est déjà une disparition. Les images de Gervais grouillent de vie comme la mort peut faire grouiller de vers le cadavre. Ce que montre avant tout ce film, c'est que la métamorphose, phénomène complexe propre au temps et à l'espace, ne se présente aucunement comme un trajet mais bien, comme son titre l'indique explicitement, comme un cycle. Le devenir relève déjà du présent. La technique et la pratique du dessin animé sont claires à cet égard: l'animateur commence par fixer les dessins-clés, non pas seulement les points de départ et d'arrivée, mais aussi les stations intermédiaires à partir desquelles les intervalles seront dessinés, dessins de raccord qui ne peuvent exister qu'en fonction du dessin-clé précédent et du dessin-clé suivant. Le bouclage, le conditionnement de l'avant par l'après, et de l'après par l'avant, préside ainsi à toute animation. Toute image animée est déjà un raccord. Cycle rend évident la continuité, l'indissociabilité voire la confusion des pôles de vie et de mort. Toutefois, c'est surtout le second film de Suzanne Gervais qui mène à terme l'idée d'un rapprochement possible entre la métamorphose animée et les thanatomorphoses du cadavre.

Climats, réalisé en 1975, tente de rendre, par le passage de la figuration à une certaine abstraction, le développement des sentiments amoureux nés de la rencontre entre les mondes intérieurs de deux individus ${ }^{25}$. C'est ici à nouveau l'impalpable, la traduction d'une réalité par l'intermédiaire de couleurs fugitives et de formes éphémères, fondues et refondues, défaites 
et refaites, qui sont au centre de la recherche picturale de Gervais. L'œuvre est construite comme un crescendo, les deux êtres étant amenés peu à peu à se rapprocher. La décomposition formelle reçoit la mission d'exprimer la métamorphose intérieure des personnages, leur évolution psychologique. Mais cet aspect narratif, cette ébauche d'intrigue, manœuvre visant à rendre le film présentable et accessible au public, doit peu nous intéresser en définitive et si les images retiennent notre attention, c'est pour une tout autre raison : involontairement ou non, peu importe, Suzanne Gervais met en lumière avec ce film, peut-être comme jamais, la prégnance de la mort dans le cinéma d'animation.

Combinant de l'encre (qui se fixe rapidement) et de l'aquarelle (qui se répand sensuellement) sur du papier, le film fait se transformer les éléments picturaux directement sous la caméra. Cette technique ne permet aucune erreur. Deux motifs sont engagés dans une telle peinture mouvante: d'une part, une série de grands paysages, infiniment ouverts et colorés; d'autre part, deux visages, parfois de profil, le plus souvent de face, immobiles, à l'expression neutre. Le film est composé d'une série de petites séquences mettant en scène de légères mais continues mutations. L'importance et la vitesse des métamorphoses ne cessent d'augmenter jusqu'à la dernière séquence. Les motifs, qu'ils soient ceux des paysages ou des visages (les deux motifs se confondent, ils fonctionnent comme des alter ego l'un pour l'autre et leur équivalence est bien entendu cruciale: ce sont en effet moins les motifs qui comptent ici que le processus de métamorphose, de retour à la matière), sont modifiés par petites touches de couleur. Ce sont principalement les tons des couleurs qui sont appelés à se modifier (du brun au bleu, du bleu au gris, du gris au blanc). Les touches se font bientôt taches. Bien qu'elles soient travaillées par Suzanne Gervais en couches superposées, en effet de recouvrement, ces taches semblent sourdre des visages-paysages mêmes, comme s'ils étaient travaillés de l'intérieur (par les vers que décrivait Philippe Ariès, justement). Le spectateur se surprend alors à scruter ces visages pour y saisir le travail du temps, pour comprendre la cohérence des germinations qui hâtent leur métamorphose, et se 
laisse fasciner par le rythme des mutations qui les transforment. La véritable virulence du film, et sa très grande force, ne se trouve pas ailleurs: ces visages disparaissant et resurgissant, effacés mais persistants, minés de toutes parts, rappellent soudain que le visage a la possibilité de connaître sa propre mort. Traitant du visage au cinéma, Jacques Aumont (1992, p. 197) avait cette révélation:

Le visage est l'apparence d'un sujet qui se sait humain, mais tous les hommes sont mortels: le visage est donc l'apparence d'un sujet qui se sait mortel. Ce qu'on cherche sur le visage, c'est le temps, mais en tant qu'il signifie la mort. La perte du visage, si perte il y a, a pour finir cette signification: elle est la mort perdue, la privation de la mort.

Le film de Suzanne Gervais ne nie pas le travail de la mort. Le visage ne s’y efface pas, il s’y abîme progressivement. Lividités éparses, corruption contaminatrice, dégradation généralisée: la mort est ici encore créatrice de formes (par ailleurs particulièrement charmantes dans le cadre de ce film: les images ne provoquent évidemment ici aucune répulsion), opérant dans le micromontage de l'animation. Oscillant entre les tons ocre et bleutés, certaines formes se gonflent, puis semblent se libérer de leur contenu coloré et se fondre dans la masse indistincte des couches d'aquarelle. Parfois, le visage retrouve une forme pure et claire, dernière réalisation d'un éphémère embaumement, avant de sombrer dans une nouvelle déliquescence. La dernière séquence du film voit les couleurs se répandre, dégouliner d'une façon tout entropique. Le visage-paysage coule, laisse ses liqueurs naturelles s'échapper, et retourne à son état premier de peinture liquide. En ce sens, le film de Suzanne Gervais n'a bien sûr rien d'abstrait, il est même sans doute l'un des films les plus matérialistes, au sens le plus technique mais surtout le plus philosophique du terme (toujours cette idée du cycle), de l'histoire du cinéma d'animation.

\section{VIII.}

Ainsi, il apparaît que le cinéma d'animation ne soit aucunement privé de l'opération du montage. D'abord parce que, on 




Photo tirée de la production Climats réalisée par Suzanne Gervais, produite par Pierre Moretti. (C) Office national du film du Canada, 1974. Tous droits réservés.

l'a dit, selon le type d'animation, de mode de fabrication et de liaison des images, le monteur est appelé à intervenir dès la préparation du film. Ensuite, parce que dans la majeure partie de sa production, le cinéma d'animation rejoue l'écriture filmique et recourt au découpage. De plus, il n'hésite pas à intégrer à sa diégèse cette énergie proprement mécanique du montage cinématographique. Térésa Faucon, dans un article surprenant ${ }^{26}$, revient sur une série de cartoons produits par 


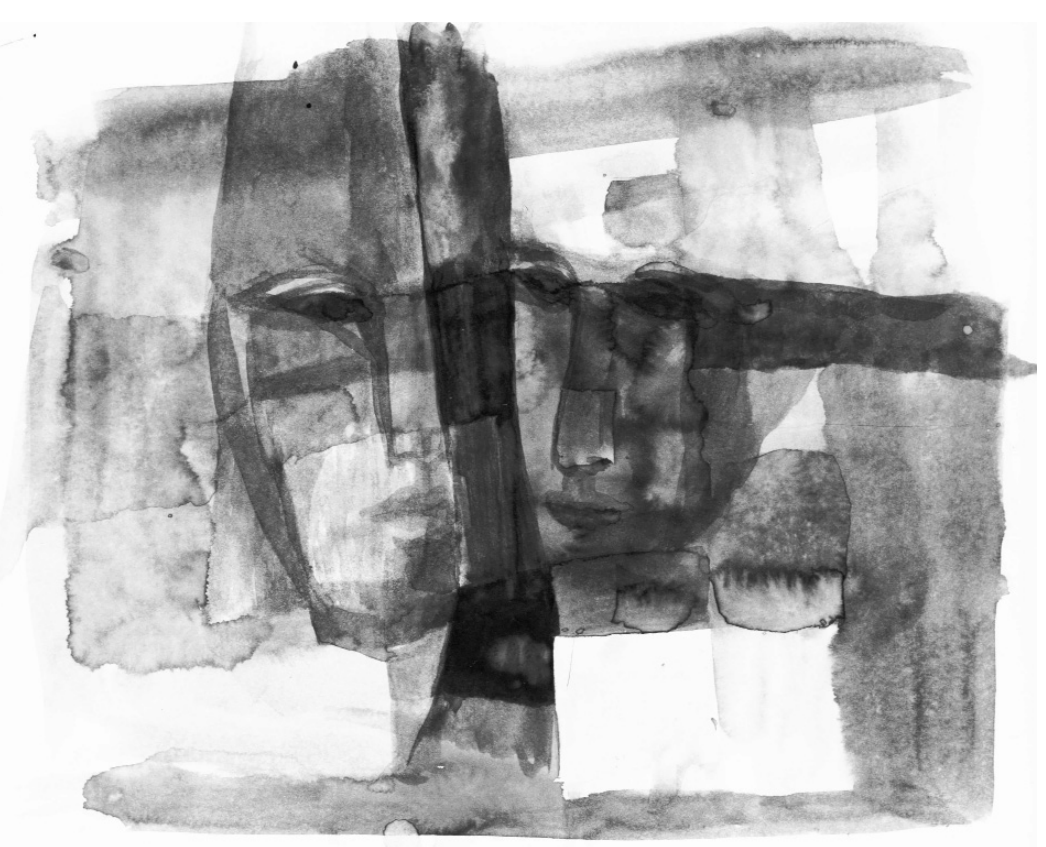

Photo tirée de la production Climats réalisée par Suzanne Gervais, produite par Pierre Moretti. () Office national du film du Canada, 1974. Tous droits réservés.

Disney, qui à travers de nombreuses références mécaniques (tuyauterie, assemblages, raccords divers de membres épars) proposent une réflexion sur le montage cinématographique en tant qu'activité de découpage et de collage, de mises à bout, d'emboîtements et d'empilements ${ }^{27}$. Cependant, et ceci explique peut-être l'obsession de certains films d'animation pour la mécanique, le montage en animation procède d'une nature plus biologique quanatomique ou mécaniste: on travaille à la génération de cellules et non à un assemblage proprement dit. Il est donc nécessaire de reconsidérer le rôle et les limites du montage dans le cinéma d'animation, relié bien plus à l'image par image qu'au plan par plan. Il ne s'agit plus ici "simplement» d'un travail de montage de plans, d'associations d'éléments épars, de collage de morceaux divers, de constitution d'un grand corps monstrueux, "frankensteinien", mais bien d'une gestion de passages, de modulations d'une image à l'autre, de transfor- 
mations d'éléments inanimés, de transmutations de figures fixes, de métamorphoses permanentes de corps inertes. En ce sens, le projet du cinéma d'animation n'est peut-être pas autant celui, fantasmatique et largement rapporté par la plupart des discours qui se risquent à le décrire, du don de vie, que celui, véritablement fantasmagorique, d'animation de la mort.

\section{Université de Liège}

\section{NOTES}

1. Ces trois étapes sont dressées notamment par Jacques Aumont, Michel Marie, Alain Bergala et Marc Vernet, dans leur ouvrage Esthétique du film (1983, p. 37-38).

2. «Pour présenter le montage, je dirai d'abord que cette opération fait partie de la dernière phase de la fabrication des films, de la post-production. Montage image, montage-son, mixage et travaux de laboratoire constituent ce que l'on appelle également les finitions" (Villain 1991, p. 7).

3. L'animateur détaille le fonctionnement et les possibilités de son invention dans un article intitulé "L'écran d'épingles" paru initialement dans Le Technicien du film, $\mathrm{n}^{\circ} 27$ et n ${ }^{\circ} 28$, recorrigé par la suite et publié par Giannalberto Bendazzi (1983).

4. Voir Sifianos 1995 (p. 25-29).

5. Voir Frierson 1994 (p. 21).

6. Voir Sifianos 1988 (p. 244). Ajoutons, afin d'éviter les écueils d'une idéologie techniciste, qu'en dépit de l'extrême variété des techniques et de l'importance que nous semblons leur accorder dans ce texte, ce sont bien entendu les images telles qu'elles apparaissent projetées qui doivent rester au centre de notre réflexion. Sur l'enjeu narratif de la métamorphose, on pourra d'ailleurs lire Paul Wells (1998, notamment p. 69-76). Pour les implications de la métamorphose dans des problématiques de représentation et de simulation, on consultera aussi les nombreux essais figurant dans l'ouvrage de référence dirigé par Alan Cholodenko, The Illusion of Life (1991).

7. On se souvient qu'au-delà de la thématique du remembrement qui traverse le concept même de montage, la mythologie frankensteinienne est déjà très présente dans les discours entourant la naissance du cinématographe, présenté comme l'appareil fantasmatique de la suppression de la mort. Voir Burch 1991 (p. 26).

8. Lire le journal de Pierre Hébert publié dans Jean 1996 (p. 221).

9. On trouvera de précieuses indications historiques quant à la production de la série dans, entre autres, Crafton 1993 (p. 167-177) et Cabarga 1988.

10. Michael Frierson (1994, p. 91) décrit le personnage de Koko le clown comme un être "amphibien", capable d'évoluer dans différents milieux. C'est particulièrement évident dans un film comme Modeling où Koko passe d'un dessin rotoscopé sur papier aux images photographiques, pour devenir ensuite une figurine de glaise et retourner enfin à une tache d'encre inanimée.

11. Il est vrai que les studios Fleischer ont l'habitude du cartoon et ne connaissent que très peu le monde de la pâte à modeler.

12. Du point de vue du langage du cinéma, le film s'inscrit encore dans cette pulsion linéaire d'établissement d'un mode de représentation institutionnel entamé 
depuis un peu plus d'une décennie et largement théorisé, entre autres, par Noël Burch.

13. Notamment en opposant la blancheur éclatante et irréelle des fonds d'images des séquences animées contre la grisaille des décors réalistes de l'atelier dans les séquences en prises de vues réelles.

14. Les motifs macabres, très en vogue dans les différents spectacles de lanterne magique, n'ont bien évidemment pas attendu Robertson pour exister. Mais ce dernier semble les avoir définitivement installés dans les images animées, que l'on pense, entre mille autres exemples, aux squelettes joyeux de la Skeleton Dance d'Ub Iwerks et Walt Disney (1929) ou à l'effroyable épouvantail de The Nightmare Before Christmas d'Henri Selick d'après l'œuvre de Tim Burton (1994).

15. Rapporté par Françoise Levie (1990, p. 79).

16. Voir Levie 1990 (p. 244).

17. On hésite à convoquer une imagerie fécale...

18. Ou du moins apparaissant comme tels.

19. Voir Thomas 2000 (p. 480-481).

20. Voir Thomas 1980.

21. Voir Thomas 1991.

22. On trouvera une description plus détaillée dans l'ouvrage du biologiste Jacques Ruffié (2000, p. 299-305).

23. Il est de fait malaisé de situer dans le temps le moment exact du trépas. "Il est vrai de dire que l'on meurt toujours progressivement, non seulement dans l'agonie mais aussi, à la limite, dans la mort subite, à la fois par degrés et par morceaux: la mort est un processus, non un état" (Thomas 1988, p. 17).

24. On peut citer ici les œuvres, entre autres, de René Jodoin, Viviane Elnécavé, Pierre Moretti, Jacques Drouin ou, tout dernièrement encore, de Michèle Cournoyer.

25. D’après le dossier de presse du film.

26. Térésa Faucon (1999) rapproche un cartoon de Disney, Donald and Pluto (1936), du film Carrie de Brian DePalma (1976).

27. The Mechanical Cow (1927), Building a Building (1933), Mickey's Mechanical Man (1933), etc. Mais on peut trouver sans peine de nombreux autres exemples de mécanisation invraisemblable dans l'histoire du cinéma d'animation. Les machines farfelues sont en effet un thème incroyablement récurrent en animation.

\section{RÉFÉRENCES BIBLIOGRAPHIQUES}

Alexeieff 1966 : Alexandre Alexeieff, Synthèse cinématographique des mouvements artificiels, Paris, IDHEC, 1966.

Amiel 2001 : Vincent Amiel, Esthétique du montage, Paris, Nathan, 2001.

Ariès 1975: Philippe Ariès, Essais sur l'histoire de la mort en Occident, Paris, Seuil, 1975.

Aumont 1992: Jacques Aumont, Du visage au cinéma, Paris, Étoile/Cahiers du cinéma, 1992.

Aumont et al. 1983 : Jacques Aumont, Michel Marie, Alain Bergala et Marc Vernet, Esthétique du film, Paris, Nathan, 1983.

Bazin 1994: André Bazin, "Un film bergsonien : le mystère Picasso ", Qu'est-ce que le cinéma, Paris, Cerf, 1994.

Bendazzi 1983: Giannalberto Bendazzi, Pages d'Alexeieff, Annecy, Atelier du cinéma d'Annecy, 1983. 
Burch 1991 : Noël Burch, La Lucarne de l'infini, Paris, Nathan, 1991.

Cabarga 1988: Leslie Cabarga, The Fleischer Story, New York, DaCapo Press, 1988.

Cholodenko 1991: Alan Cholodenko, The Illusion of Life, Sidney, Power Publications, 1991.

Crafton 1993: Donald Crafton, Before Mickey, Chicago/London, The University of Chicago Press, 1993.

Dovnikovic 2000 : Borivoj Dovnikovic, La Technique du dessin animé, Paris, Dreamland, 2000.

Drouin 1982: Jacques Drouin, "La spécificité et le rôle du monteur de films d'animation ", Copie Zéro, no 14, 1982.

Faucon 1999: Térésa Faucon, "Dessine-moi un raccord", Cinémathèque, n 15, 1999, p. 72-79.

Frierson 1994: Michael Frierson, Clay Animation, New York, Twayne Publishers, 1994.

Jean 1996: Marcel Jean, Pierre Hébert, l'homme animé, Montréal, Les 400 coups, 1996.

Levie 1990 : Françoise Levie, Étienne-Gaspard Robertson, la vie d'un fantasmagore, Bruxelles/Longueuil, Sofidoc/Les Éditions du Préambule, 1990.

Ruffié 2000 : Jacques Ruffié, Le Sexe et la mort, Paris, Odile Jacob, 2000.

Sifianos 1988: Georges Sifianos, Langage et esthétique du cinéma d'animation, thèse de doctorat, Paris, Université de Paris I, 1988.

Sifianos 1995: Georges Sifianos, «Entrevue avec Peter Lord», Positif, n 407, 1995, p. 25-29.

Thomas 1980 : Louis-Vincent Thomas, Le Cadavre. De la biologie à l'anthropologie, Bruxelles, Complexe, 1980.

Thomas 1988: Louis-Vincent Thomas, La Mort, Paris, PUF, 1988.

Thomas 1991 : Louis-Vincent Thomas, La Mort en question, Paris, L'Harmattan, 1991.

Thomas 2000 : Louis-Vincent Thomas, Les Chairs de la mort, Paris, Institut d'édition, 2000.

Villain 1991 : Dominique Villain, Le Montage au cinéma, Paris, Cahiers du cinéma, 1991.

Wells, 1998 : Paul Wells, Understanding Animation, New York, Routledge, 1998. 